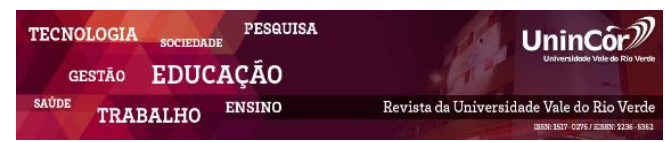

Revista da Universidade Vale do Rio Verde ISSN: 1517-0276 / EISSN: 2236-5362 v. 17 | n. 1 | Ano 2019

Luciene da Silva Castro

Universidade Federal de Minas Gerais lucienecasttro@hotmail.com

Audrei Giménez Barañano

Universidade Federal do Espírito Santo audrei3@gmail.com

\title{
PREDIÇÃO DE PARÂMETROS DE QUALIDADE DO BIODIESEL DE DIFERENTES OLEAGINOSAS
}

\section{RESUMO}

O biodiesel é uma mistura de ésteres monoalquílicos de ácidos graxos de cadeia longa, derivado a partir de biomassa tais como óleos vegetais e gordura animal. A caracterização do biodiesel depende da composição da matéria-prima de origem. A densidade, a viscosidade, o número de cetano e o calor de combustão são parâmetros que avaliam a qualidade do biodiesel como combustível. O objetivo do presente artigo consiste em predizer a densidade, a viscosidade, o calor de combustão e o índice de cetano do biodiesel de diferentes oleaginosas através de equações empíricas. O biodiesel de óleo de semente de maracujá é mais denso comparado aos demais biodieseis, isso se deve ao fato desse biodiesel ser constituído de ésteres de ácidos graxos insaturados, enquanto o biodiesel de gordura suína é o mais viscoso comparado aos demais biodieseis em estudo, devido a gordura suína ser composta basicamente por ácidos graxos de cadeia longa. O biodiesel de gordura suína apresentou maior calor de combustão, isso se deve ao número de carbonos presentes na molécula desse biodiesel. O biodiesel de óleo de palma apresentou maior índice de cetano, o que se deve à saturação e ao comprimento da cadeia carbônica de ácidos graxos. O número de cetano, a viscosidade e o calor de combustão aumentam devido ao aumento da massa molar e diminuem à medida que o número de ligações duplas aumenta, enquanto a densidade aumenta com a diminuição da massa molecular e com o aumento do grau de insaturação.

Palavras-chave: Predição. Densidade. Viscosidade. Índice de Cetano. Calor de combustão.

\section{QUALITY PARAMETERS PREDICTION OF BIODIESEL FROM DIFFERENT SEED OILS}


saturation and carbonic acid chain length of fatty acids. The number of cetane, viscosity and heat of combustion increase due to the increase in molar mass and decrease as the number of double bonds increases while the density increases with decreasing molecular mass and with increasing unsaturation degree.

Keywords: Prediction. Density. Viscosity. Cetane Number. High Heat Value.

Recebido em: 03/09/2018 - Aprovado em: 25/03/2019 - Disponibilizado em: 15/07/2019

\section{INTRODUÇÃO}

O biodiesel é um importante combustível e promissor substituto ao diesel, pois é um combustível renovável, biodegradável, apresenta baixa toxicidade e baixa emissão de gases poluentes, sendo considerado um combustível limpo ou verde (KAFUKU; MBARAWA, 2010).

O biodiesel é uma mistura de ésteres monoalquílicos de ácidos graxos de cadeia longa, derivado a partir de biomassa tais como óleos vegetais e gordura animal (DEMIRBAS, 2009).

A escolha da matéria-prima é primeiro passo para produção de biodiesel (KARMAKAR; KARMAKAR; MUKHERJEE, 2010), pois é responsável pelo maior custo desse processo (ATADASHI; AROUA; AZIZ, 2010). De acordo com Silitonga et al (2011), a matériaprima para produção de biodiesel é classificada em:

- Óleo vegetal comestível: soja, palma, girassol, milho e canola;

- Óleo vegetal não comestível: pinhão manso, semente de seringueira e algas;

- Óleo reciclado ou resíduo;

- Gordura animal: graxa amarela, gordura de frango, sebo bovino e óleo de peixe.

As propriedades físico-químicas do biodiesel são determinadas pelos perfis de composição da matéria-prima de origem, podendo, assim variar muito conforme a matériaprima utilizada na sua produção (HOEKMAN et $a l, 2012)$. Entre essas propriedades estão a densidade, a viscosidade, o índice de cetano e o poder calorífico, que são parâmetros que avaliam a qualidade do biodiesel como combustível.

A densidade e viscosidade tem efeito direto sobre o processo de atomização durante a combustão. A densidade sempre está relacionada à estrutura da matéria-prima e, normalmente, o aumento da massa molecular conduz ao aumento da densidade. A viscosidade está relacionada ao tamanho das cadeias carbônicas dos ésteres, ou seja, o aumento do número de carbonos e do grau de saturação leva ao aumento da viscosidade. Altas viscosidades dos biodieseis levam a diminuição da eficiência da combustão, aumentando o depósito de resíduos no motor (RAMÍREZ-VERDUZCO; RODRÍGUEZRODRÍGUEZ; JARAMILLO-JACOB, 2012).

O calor de combustão é a quantidade de calor liberada durante a combustão de um grama de combustível para produzir $\mathrm{CO}_{2}$ e $\mathrm{H}_{2} \mathrm{O}$ na sua temperatura inicial (RAMÍREZ-VERDUZCO; RODRÍGUEZ-RODRÍGUEZ; JARAMILLOJACOB, 2012).

O número de cetano é responsável pela avaliação da qualidade de injeção do 
combustível, ou seja, relaciona-se com o retardo de ignição do combustível de modo que, quanto menor o número de cetano, maior o retardo. Portanto, um número de cetano baixo, não é desejável em virtude da pequena queima de combustível no tempo certo será baixa, assim dificultando o funcionamento do motor. A especificação brasileira não define valores para o biodiesel. Na Europa, o número de cetano mínimo aceitável foi fixado em 51 para o biodiesel, pelo método EN ISO 5165, enquanto os EUA exigem 47 para o biodiesel pelo método D 613 (LÔBO; FERREIRA; CRUZ, 2009). O número de cetano médio do biodiesel produzido no Brasil é 60. A combustão do biodiesel é mais eficiente em motor diesel do que a combustão do próprio diesel mineral (REVISTA BIODIESEL, 2008).

O objetivo do presente artigo consiste em predizer a densidade, viscosidade, o calor de combustão e o índice de cetano do biodiesel de diferentes oleaginosas através de equações empíricas.

\section{METODOLOGIA}

As propriedades dos biodieseis tais como: densidade, viscosidade, calor de combustão e índice de cetano foram preditas pelas equações empíricas propostas por RamírezVerduzco; Rodríguez-Rodríguez; JaramilloJacob (2012). Em todas as correlações empíricas, $M_{i}$ corresponde à massa molecular dos ésteres metílicos de ácidos graxos e $N$ é o número de ligações insaturadas presentes nos ésteres metílicos de ácidos graxos.

A densidade à $20^{\circ} \mathrm{C}$ do biodiesel, $\rho_{b}$, foi predita pela equação 1 , expressa $\mathrm{em} \mathrm{g} / \mathrm{cm}^{3}$. Onde $w_{i}$ é a fração mássica e $\rho_{i}$ é a densidade dos ésteres metílicos de ácidos graxos em $\mathrm{g} / \mathrm{cm}^{3}$ obtida pela equação 2 .

$$
\begin{aligned}
& \rho_{b}=\sum_{i=1}^{n} w_{i} \rho_{i} \\
& \rho_{i}=0,8453+\frac{4,9}{M_{i}}+0,0118 . N
\end{aligned}
$$

A viscosidade à $40^{\circ} \mathrm{C}$ do biodiesel, $\eta_{b}$, expressa $\mathrm{em} \mathrm{mm}^{2} / \mathrm{s}$ foi predita pela equação 3 . Onde $\eta_{i}$ é a viscosidade à $40^{\circ} \mathrm{C}$ dos ésteres metílicos de ácidos graxos obtida a partir da equação 4 .

$\eta_{b}=\sum_{i=1}^{n} w_{i} \eta_{i}$

$\ln \left(\mathrm{\eta}_{i}\right)=-12,503+2,496 \ln \left(M_{i}\right)-0,178 N(4)$

O calor de combustão do biodiesel, $\delta_{b}$, expresso em $\mathrm{MJ} / \mathrm{kg}$ foi predito pela equação 5 . O calor de combustão dos ésteres metílicos de ácidos graxos, $\delta_{i}$, em $\mathrm{MJ} / \mathrm{kg}$, foi obtido pela equação 6.

$$
\begin{aligned}
& \delta_{b}=\sum_{i=1}^{n} w_{i} \delta_{i} \\
& \delta_{i}=46,19-\frac{1794}{M_{i}}-0,21 N
\end{aligned}
$$

O índice de cetano do biodiesel, $\emptyset_{b}$, foi predito pela equação 7 , onde $\emptyset_{i}$ é o índice de cetano dos ésteres metílicos de ácidos graxos, obtido pela equação 8 .

$$
\begin{aligned}
& \emptyset_{b}=\sum_{i=1}^{n} w_{i} \emptyset_{i} \\
& \emptyset_{i}=-7,8+0,302 M_{i}-20 N
\end{aligned}
$$

A fração molar $\left(x_{i}\right)$ foi estimada para cada éster metílico a partir da composição mássica, através da equação 9. A massa molecular $(\bar{M})$ foi estimada pela equação 10 . 
$x_{i}=\frac{\frac{w_{i}}{M_{i}}}{\sum_{i=1}^{n} \frac{w_{i}}{M_{i}}}$

$\bar{M}=\sum_{i=1}^{n} x_{i} \cdot M_{i}$

A composição de ácidos graxos das oleaginosas em estudo foi obtida a partir de Allen et al (1999); Chammoun, Geller e Das (2013); Koh e Tinia (2011); Navarro-Díaz et al (2014); Roschat et al (2017); Silva et al., (2014); Yahyaee, Ghobadian e Najafi (2013).

\section{RESULTADOS E DISCUSSÕES}

A Tabela 1 apresenta a composição mássica ésteres metílicos de gordura suína, ésteres metílicos de óleo de palma, ésteres metílicos de óleo de nabo forrageiro, ésteres metílicos de óleo de pinhão manso, ésteres metílicos de óleo de macaúba, ésteres metílicos de óleo de babaçu, ésteres metílicos de óleo de semente de seringueira, ésteres metílicos de óleo de coco, ésteres metílicos de óleo de semente de maracujá.

Tabela 1 - Frações mássicas dos biodieseis de diferentes oleaginosas.

\begin{tabular}{|c|c|c|c|c|c|c|c|}
\hline \multirow{2}{*}{$\begin{array}{c}\text { Ester } \\
\text { metílico }\end{array}$} & \multicolumn{7}{|c|}{$w_{i}$} \\
\hline & $\begin{array}{c}\text { Gordura } \\
\text { Suína } \\
\end{array}$ & Palma & $\begin{array}{l}\text { Pinhão } \\
\text { Manso }\end{array}$ & Babaçu & $\begin{array}{l}\text { Semente de } \\
\text { Seringueira }\end{array}$ & Coco & $\begin{array}{c}\text { Semente de } \\
\text { maracujá }\end{array}$ \\
\hline C6:0 & - & - & - & - & - & - & - \\
\hline C8:0 & - & 0,0010 & - & 0,0520 & - & 0,0830 & - \\
\hline C10:0 & 0,0010 & 0,0010 & - & 0,0480 & - & 0,060 & - \\
\hline C12:0 & 0,0010 & 0,0090 & - & 0,5180 & - & 0,4670 & - \\
\hline C14:0 & 0,0140 & 0,0130 & - & 0,2220 & - & 0,1830 & - \\
\hline C16:0 & 0,2550 & 0,4390 & 0,1420 & 0,0320 & 0,0967 & 0,0920 & 0,0973 \\
\hline C16:1 & - & - & 0,0140 & - & - & - & 0,0011 \\
\hline C17:0 & - & - & - & - & - & - & - \\
\hline C17:1 & - & - & - & - & - & - & - \\
\hline C18:0 & 0,1580 & 0,0490 & 0,0690 & 0,0330 & 0,0556 & 0,0290 & 0,0258 \\
\hline C18:1 & 0,4710 & 0,3900 & 0,4310 & 0,0730 & 0,2385 & 0,0690 & 0,1383 \\
\hline C18:2 & 0,0890 & 0,0950 & 0,3440 & 0,0030 & 0,4591 & 0,0170 & 0,7314 \\
\hline C18:3 & 0,0110 & 0,0030 & - & - & 0,1411 & - & 0,0041 \\
\hline C20:0 & - & - & - & - & - & - & 0,0010 \\
\hline C20:1 & - & - & - & - & - & - & 0,0010 \\
\hline
\end{tabular}

O estereato de metila é o principal componente presente no biodiesel de gordura de suína, ou seja, corresponde a quase metade dos ésteres metílicos presentes, enquanto o biodiesel de óleo de palma é composto principalmente pelo éster palmitato de metila, que juntamente com o éster oleato de metila corresponde a $83 \%$ do biodiesel.

O biodiesel de pinhão manso é constituído principalmente do éster metílicos insaturados tais como o oleato de metila e linoleato de metila. Ambos são ésteres de cadeia longa e representam 77,5\% dos ésteres presentes no biodiesel. Já o biodiesel proveniente do óleo de babaçu é formado por ésteres de cadeia média tais como laurato de metila e miristato de metila.

Os dois principais ésteres de metila presentes no biodiesel de semente de seringueira são semelhantes ao biodiesel de pinhão manso, o mais representativo é o éster linoleato de metila, . 
A composição do biodiesel de óleo de coco é semelhante ao biodiesel de óleo de babaçu, constituído principalmente por ésteres de ácidos graxos saturados e de cadeia carbônica média. O linoleato de metila corresponde quase
3/4 dos ésteres metílicos do óleo de semente de maracujá.

A Tabela 2 mostra a fração molar dos biodieseis de diferentes oleaginosas. Observa-se que a fração molar é semelhante aos valores da fração mássica.

Tabela 2 - Fração molar dos biodieseis de diferentes oleaginosas.

\begin{tabular}{|c|c|c|c|c|c|c|c|}
\hline \multirow{2}{*}{$\begin{array}{c}\text { Ester } \\
\text { metílico }\end{array}$} & \multicolumn{7}{|c|}{$x_{i}$} \\
\hline & $\begin{array}{l}\text { Gordura } \\
\text { Suína }\end{array}$ & Palma & $\begin{array}{l}\text { Pinhão } \\
\text { Manso }\end{array}$ & Babaçu & $\begin{array}{l}\text { Semente de } \\
\text { Seringueira }\end{array}$ & Coco & $\begin{array}{l}\text { Semente de } \\
\text { maracujá }\end{array}$ \\
\hline C6:0 & - & - & - & - & - & - & - \\
\hline C8:0 & - & 0,0018 & - & 0,0746 & - & 0,1164 & - \\
\hline C10:0 & 0,0015 & 0,0015 & - & 0,0585 & - & 0,0714 & - \\
\hline C12:0 & 0,0013 & 0,0119 & - & 0,5487 & - & 0,4833 & - \\
\hline C14:0 & 0,0167 & 0,0151 & - & 0,2080 & - & 0,1675 & - \\
\hline C16:0 & 0,2718 & 0,4582 & 0,1531 & 0,0269 & 0,1055 & 0,0755 & 0,1052 \\
\hline C16:1 & - & - & 0,0152 & - & - & - & 0,0012 \\
\hline C17:0 & - & - & - & - & - & - & - \\
\hline C17:1 & - & - & - & - & - & - & - \\
\hline C18:0 & 0,1526 & 0,0463 & 0,0674 & 0,0251 & 0,0550 & 0,0216 & 0,0253 \\
\hline C18:1 & 0,4580 & 0,3713 & 0,4238 & 0,0559 & 0,2373 & 0,0516 & 0,1364 \\
\hline C18:2 & 0,0871 & 0,0911 & 0,3406 & 0,0023 & 0,4599 & 0,0128 & 0,7261 \\
\hline C18:3 & 0,0108 & 0,0029 & - & - & 0,1423 & - & 0,0041 \\
\hline C20:0 & - & - & - & - & - & - & 0,0009 \\
\hline C20:1 & - & - & - & - & - & - & 0,0009 \\
\hline
\end{tabular}

A massa molecular do biodiesel de óleo de semente de seringueira, Tabela 3, apresentou maior massa molecular em virtude ser constituído principalmente por ésteres de ácidos graxos de cadeia longa.

Tabela 3 - Massa molecular dos biodieseis.

\begin{tabular}{lc}
\hline Biodiesel & $\mathbf{M}_{\text {calculada }}(\mathbf{g} / \mathbf{m o l})$ \\
\hline Gordura suína & 288,3163 \\
Palma & 282,2512 \\
Pinhão manso & 291,5253 \\
Babaçu & 222,7480 \\
Semente de seringueira & 292,3518 \\
Coco & 221,8240 \\
Semente de maracujá & 292,3388 \\
\hline
\end{tabular}

A densidade, a viscosidade, o índice de cetano e o calor de combustão, preditos e reportados na literatura, estão representados na Tabela 4.

A densidade predita está na faixa de 0,8527 - 0,8821. A elevada densidade do biodiesel de óleo de semente de maracujá comparada aos demais biodieseis deve ao fato desse biodiesel ser constituído por $84,5 \%$ de ésteres de ácidos graxos insaturados. O grau de insaturação influencia diretamente à densidade do biodiesel. Todas as densidades preditas estão de acordo a especificação dos padrões de qualidade do biodiesel, pois a mesma deve está na faixa 850 a $900 \mathrm{~kg} / \mathrm{m}^{3}$ de acordo com o Regulamento técnico ANP no 03/2012. 
Segundo o Regulamento técnico ANP $n^{\circ}$ $03 / 2012$, a viscosidade à $40^{\circ} \mathrm{C}$ deve estar na faixa 3,0 a $6,0 \mathrm{~mm}^{2} / \mathrm{s}$. Observa-se, na Tabela 4, que todos os biodieseis estão de acordo com a especificação dos padrões de qualidade do biodiesel, exceto o biodiesel de óleo de coco e biodiesel de óleo de babaçu, pois ambos apresentam uma baixa viscosidade. Essa baixa viscosidade se deve ao fato de ambos apresentarem uma baixa massa molecular, comparado aos demais biodieseis, e por serem constituídos principalmente de ácidos graxos saturados. A maior viscosidade encontrada entre os biodieseis foi para o biodiesel de gordura suína, devido à gordura suína ser composta basicamente por ácidos graxos de cadeia longa.

O calor de combustão dos biodieseis obtidos foi muito próximo. O biodiesel de gordura suína apresentou maior calor de combustão, isto é, apresentou maior liberação de energia durante a combustão, isso se deve ao número de carbonos presentes na molécula desse biodiesel.

O número de cetano variou entre 48 a 65 . $\mathrm{O}$ biodiesel de óleo de palma apresentou maior índice de cetano, isso evidencia que melhor será sua a combustão em motor, pois o número de cetano é inversamente proporcional ao retardo da ignição, ou seja, maior número de cetano implica em menor retardo da ignição. Esse valor se deve à saturação e ao comprimento da cadeia carbônica de ácidos graxos. Nota-se ainda que biodiesel de óleo de seringueira apresentou menor número de cetano, isso ocorre devido ao número de insaturações presentes.

\section{CONCLUSÃO}

Os parâmetros de qualidade dos biodieseis de diferentes oleaginosas foram preditos a partir de equações empíricas e foi possível observar a semelhança com os parâmetros obtidos a partir de experimentos laboratoriais.

O biodiesel de óleo de semente de maracujá apresentou maior densidade comparado ao biodiesel de gordura suína é o mais viscoso comparado aos demais biodieseis estudados, devido à gordura suína ser composta basicamente por ácidos graxos de cadeia longa.

O biodiesel de gordura suína apresentou maior calor de combustão, isto é, apresentou maior liberação de energia durante a combustão, isso se deve ao número de carbonos presentes na molécula desse biodiesel.

O biodiesel de óleo de palma apresentou maior índice de cetano, isso evidencia que melhor será sua a combustão em motor, se deve a saturação e ao comprimento da cadeia carbônica de ácidos graxos.

\section{AGRADECIMENTOS}

Os autores agradecem a Fundação de Amparo à Pesquisa e Inovação do Espírito Santo (FAPES) pela concessão da bolsa de mestrado. 
Tabela 4 - Propriedades do biodiesel.

\begin{tabular}{|c|c|c|c|c|c|c|c|c|}
\hline Biodiesel & $\begin{array}{c}\rho_{b_{\text {calculado à } 20^{\circ} \mathrm{C}}}\left(\mathrm{g} / \mathrm{cm}^{3}\right) \\
\end{array}$ & $\begin{array}{c}\rho_{b_{\text {reportado }}} \\
\left(\mathrm{g} / \mathrm{cm}^{3}\right)\end{array}$ & 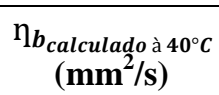 & $\begin{array}{c}\eta_{b_{\text {reportado }}} \\
\left(\mathbf{m m}^{2} / \mathbf{s}\right)\end{array}$ & $\begin{array}{c}\boldsymbol{\delta}_{\boldsymbol{b}_{\text {calculado }}} \\
(\mathbf{M J} / \mathbf{k g})\end{array}$ & $\begin{array}{c}\delta_{b_{\text {reportado }}} \\
(\mathbf{M J} / \mathbf{k g})\end{array}$ & $\emptyset_{b_{\text {calculado }}}$ & $\emptyset_{b_{\text {reportado }}}$ \\
\hline $\begin{array}{l}\text { Gordura } \\
\text { Suína }\end{array}$ & 0,8713 & $\begin{array}{l}0,8730^{\mathrm{B}, \mathrm{a}} \\
0,8371^{\mathrm{B}, \mathrm{b}}\end{array}$ & 4,5545 & $\begin{array}{l}5,0800^{\mathrm{a}} \\
2,4910^{\mathrm{b}}\end{array}$ & 39,8244 & $\begin{array}{l}40,1000^{\mathrm{a}} \\
38,8000^{\mathrm{b}}\end{array}$ & 65,8360 & $57,8000^{b}$ \\
\hline Palma & 0,8706 & $0,8644^{\mathrm{C}, \mathrm{d}}$ & 4,3984 & $\begin{array}{l}4,5^{\mathrm{c}} \\
4,33^{\mathrm{h}}\end{array}$ & 39,7103 & $41.24^{\mathrm{m}}$ & 65,9650 & $\begin{array}{c}61^{\mathrm{c}} \\
54,6^{\mathrm{d}}\end{array}$ \\
\hline $\begin{array}{l}\text { Pinhão } \\
\text { Manso }\end{array}$ & 0,8765 & $\begin{array}{c}0.8636^{\mathrm{e}} \\
0,8795^{\mathrm{B}, \mathrm{d}}\end{array}$ & $4,3139^{\mathrm{d}}$ & $\begin{array}{c}4,78^{\mathrm{e}} \\
4,8^{\mathrm{d}}\end{array}$ & 39,7982 & $\begin{array}{c}38.5^{\mathrm{e}} \\
39,23^{\mathrm{d}}\end{array}$ & 57,6767 & $51,6^{\mathrm{d}}$ \\
\hline Babaçu & 0,8527 & $0.8790^{\mathrm{f}}$ & 2,6717 & $3,239^{\mathrm{d}}$ & 37,3949 & $\begin{array}{c}9440 \\
\mathrm{kcal} / \mathrm{kg}^{\mathrm{p}}\end{array}$ & 58,1278 & $63^{f}$ \\
\hline $\begin{array}{c}\text { Semente } \\
\text { de } \\
\text { seringueira }\end{array}$ & 0,8740 & $\begin{array}{c}0,8800^{\mathrm{B}, \mathrm{h}} \\
0,8740^{\mathrm{k}}\end{array}$ & 3,9506 & $\begin{array}{l}4,84^{\mathrm{h}} \\
3,12^{\mathrm{j}}\end{array}$ & 39,3635 & $\begin{array}{r}36,5^{\mathrm{d}} \\
38.20^{\mathrm{j}}\end{array}$ & 48,2296 & $\begin{array}{l}44,2^{\mathrm{k}} \\
66,2^{\mathrm{n}}\end{array}$ \\
\hline Coco & 0,8696 & $\begin{array}{r}0,8073^{\mathrm{B}, \mathrm{d}} \\
0,8748^{\mathrm{B}, \mathrm{i}}\end{array}$ & 2,7052 & $2.937^{\mathrm{i}}$ & 38,0808 & $38.1^{\mathrm{i}}$ & 58,9267 & $46^{\circ}$ \\
\hline $\begin{array}{l}\text { Semente } \\
\text { de } \\
\text { maracujá }\end{array}$ & 0,8821 & $0,8650^{\mathrm{C}, \mathrm{l}}$ & 3,9844 & $3.9^{1}$ & 39,7140 & $31,82^{1}$ & 48,2379 & - \\
\hline
\end{tabular}

${ }^{\mathrm{A}}$ Densidade à $20^{\circ} \mathrm{C}$

${ }^{\mathrm{C}}$ Densidade à $25^{\circ} \mathrm{C}$

${ }^{\mathrm{D}}$ Densidade à $30^{\circ} \mathrm{C}$

a (MATA et al, 2010)

b(JANCHIV;OH ; CHOI, 2012)

${ }^{\mathrm{c}}$ (RAMOS et al, 2009)

d (ATABANI et al, 2012)

e (LEUNG; WU; LEUNG, 2010)

f (FUKUDA; KOND ; NODA, 2001)

$\mathrm{g}$ (MORSHED et al, 2011)

${ }^{\mathrm{B}}$ Densidade à $15^{\circ} \mathrm{C}$

h (ROSCHAT et al, 2017)

i (NAKPONG; WOOTTHIKANOKKHAN, 2010) $\mathrm{j}$ (MELVIN JOSE et al, 2011)

k (RAMADHAS; JAYARAJ; MURALEEDHARAN, 2005)

${ }^{1}$ (TIPPAYAWONG; CHUMJAI, 2012)

(DEMIRBAS, 2008)

n (GIMBUN et al, 2012)

o (OLUBUNMI, 2016)

$\mathrm{p}$ (ZUNIGA et al, 2011) 


\section{REFERÊNCIAS}

Allen CAW, Watts KC, Ackman RG, Pegg MJ. Predicting the viscosity of biodiesel fuels from their fatty acid ester composition. Fuel. 1999;78(11):13191326.

Atabani AE, Silitonga AS, Badruddin IA, Mahlia TMI, Masjuki HH, Mekhilef S. A comprehensive review on biodiesel as an alternative energy resource and its characteristics. Renew Sustain Energy Rev . 2012;16(4):2070-2093.

Atadashi IM, Aroua MK, Aziz AA. High quality biodiesel and its diesel engine application: A review. Renew Sustain Energy Rev. 2010;14(7):1999-2008.

Chammoun N, Geller DP, Das KC. Fuel properties, performance testing and economic feasibility of Raphanus sativus (oilseed radish) biodiesel. Ind Crops Prod . 2013;45:155-159.

Demirbas A. Progress and recent trends in biodiesel fuels. Energy Convers Manag. 2009;50(1):14-34.

Demirbas A. Relationships derived from physical properties of vegetable oil and biodiesel fuels. Fuel. 2008;87(8-9):1743-1748.

Fukuda H, Kond A, Noda H. Biodiesel Fuel Production by Transesterification. Biosci Bioeng. 2001;92(5):405416.

Gimbun J, Ali S, Charan Kanwal CCS, Shah LA, Ghazali NHM, Cheng CK, et al. Biodiesel Production from Rubber Seed Oil Using A Limestone Based Catalyst. Adv Mater Phys Chem. 2012;02(04):138141.

Hoekman SK, Broch A, Robbins C, Ceniceros E, Natarajan M. Review of biodiesel composition, properties, and specifications. Renew Sustain Energy Rev. 2012;16(1):143-169.

Janchiv A, Oh Y, Choi S. High quality biodiesel production from pork lard by high solvent additive. ScienceAsia. 2012;38(1):95-101.

Kafuku G, Mbarawa M. Biodiesel production from Croton megalocarpus oil and its process optimization. Fuel. 2010;89(9):2556-2560.

Karmakar A, Karmakar S, Mukherjee S. Properties of various plants and animals feedstocks for biodiesel production. Bioresour Technol. 2010;101(19):72017210 .

Koh MY, Tinia TI. A review of biodiesel production from Jatropha curcas L. oil. Renew Sustain Energy Rev. 2011;15(5):2240-2251.

Leung DYC, Wu X, Leung MKH. A review on biodiesel production using catalyzed transesterification. Appl Energy. 2010;87(4):1083-1095.

Lôbo I, Ferreira S, Cruz R. Biodiesel: quality parameters and analytical methods. Quim Nova . 2009;32(6):1596-1608.

Mata TM, Cardoso N, Ornelas M, Neves S, Caetano NS. Sustainable production of biodiesel from tallow, lard and poultry fat and its quality evaluation. Chem Eng Trans . 2010;19:13-18.

Melvin Jose DF, Edwin Raj R, Durga Prasad B, Robert Kennedy Z, Mohammed Ibrahim A. A multi-variant approach to optimize process parameters for biodiesel extraction from rubber seed oil. Appl Energy. 2011;88(6):2056-63.

Morshed M, Ferdous K, Khan MR, Mazumder MSI, Islam MA, Uddin MT. Rubber seed oil as a potential source for biodiesel production in Bangladesh. Fuel. 2011;90(10):2981-2986.

Nakpong P, Wootthikanokkhan S. High free fatty acid coconut oil as a potential feedstock for biodiesel production in Thailand. Renew Energy.

2010;35(8):1682-1687.

Navarro-Díaz HJ, Gonzalez SL, Irigaray B, Vieitez I, Jachmanián I, Hense H, et al. Macauba oil as an alternative feedstock for biodiesel: Characterization and ester conversion by the supercritical method. J Supercrit Fluids. 2014;93:130-137.

Olubunmi FA. Evaluation of Coconut Oil Biodiesel Fuels as Sustainable Alternatives to Petro-Diesel in Nigeria. Int J Sci Eng Res. 2016;7(7):574-582.

Ramadhas AS, Jayaraj S, Muraleedharan C. Characterization and effect of using rubber seed oil as fuel in the compression ignition engines. Renew Energy. 2005;30(5):795-803.

Ramírez-Verduzco LF, Rodríguez-Rodríguez JE, Jaramillo-Jacob ADR. Predicting cetane number, kinematic viscosity, density and higher heating value of biodiesel from its fatty acid methyl ester composition. Fuel. 2012;91(1):102-111.

Ramos MJ, Fernández CM, Casas A, Rodríguez L, Pérez Á. Influence of fatty acid composition of raw materials on biodiesel properties. Bioresour Technol. 2009;100(1):261-268.

Roschat W, Siritanon T, Yoosuk B, Sudyoadsuk T, Promarak V. Rubber seed oil as potential non-edible feedstock for biodiesel production using heterogeneous catalyst in Thailand. Renew Energy. 2017;101:937944.

Silitonga AS, Atabani AE, Mahlia TMI, Masjuki HH, Badruddin IA, Mekhilef S. A review on prospect of Jatropha curcas for biodiesel in Indonesia. Renew 
Sustain Energy Rev [Internet]. 2011;15(8):3733-3756.

Silva MCD, Da Silva LM, Brandão KS, Souza AG, Cardoso LP, Dos Santos AO. Low temperature properties of winterized methyl babassu biodiesel. J Therm Anal Calorim. 2014;115(1):635-640.

Tippayawong N, Chumjai P, Preparation AS.

Characterization and Performance of Biofuel from

Passion Fruit Processing Residues. 2012;2:24-27.

Yahyaee R, Ghobadian B, Najafi G. Waste fish oil biodiesel as a source of renewable fuel in Iran. Renew Sustain Energy Rev. 2013;17(6):312-319.

Zuñiga ADG, Paula MM, Coimbra JSR, Martins ECA, Silva DX, Telis-Romero J. Revisão :

propriedades físico-químicas do biodiesel. 2011;21:5572.

\section{Luciene da Silva Castro}

Doutoranda em Engenharia Química pela Universidade Federal de Minas Gerais, Mestre em Engenharia Química pela Universidade Federal do Espirito Santo e Engenheira Química pelas Faculdades Integradas de Aracruz.

\section{Audrei Giménez Barañano}

Prof $^{a}$ do Curso de Engenharia Química e Prof ${ }^{a}$ do Programa de Pós-graduação em Engenharia Química da Universidade Federal do Espírito Santo, Campus Alegre. 